\title{
Organisation von Hochschule mitgestalten
}

\author{
Studierendenpartizipation als zentrale Perspektive
}

\section{Zwischen Gestaltungswillen und Lähmung}

Organisationskultur von Hochschulen aus Studierendenperspektive zu beschreiben, gestaltet sich schwierig. Denn es gibt diese eine „Studierendenperspektive“ nicht. Die Studienmöglichkeiten differenzieren sich in Deutschland immer weiter aus. So vielfältig wie die Studienmöglichkeiten gestalten sich auch die Blickwinkel auf das Hochschulsystem. Trotz der starken Ausdifferenzierung haben die meisten Studierenden die intensivste Begegnung mit der Hochschule in ihren Lehrveranstaltungen. Zu konzeptioneller Arbeit im Kontext von Hochschulentwicklung fühlen sich die meisten Studierenden eher nicht berufen. Dies zeigt ein Blick auf die Wahlbeteiligungen bei Gremienwahlen in Hochschulen. Diese liegen meistens im einstelligen Bereich. Doch für die Entwicklung von Hochschulen ist es wichtig, motivierte Studierende so intensiv wie möglich aktiv in strukturelle Verantwortung einzubinden. Dadurch wird auch der Kontakt zur Studierendenschaft hergestellt. Dies ist von besonderer Relevanz, denn Studierende bringen als Hauptnutzendengruppe der Hochschule einen wichtigen Blick auf die Entwicklungsnotwendigkeiten mit. Der folgende Beitrag basiert auf einer individuellen Perspektive auf das Hochschulsystem und begrenzt sich damit nur auf einen Ausschnitt des deutschen Hochschulsystems. Der Fokus liegt dabei auf der Situation in Baden-Württemberg. Die Thesen sind in Zusammenarbeit mit den Mitgliedern der Arbeitsgruppe \#DigitalChangeMaker, einer studentischen Arbeitsgruppe des Hochschulforums Digitalisierung, entstanden.

Der vorliegende Beitrag soll in drei Schritten für eine stärkere Einbindung der Studierenden in die strukturellen und strategischen Entscheidungen der Hochschulen argumentieren. Zuerst werden anhand des baden-württembergischen Landeshochschulgesetzes die verschiedenen Entscheidungsebenen an der Hochschule dargestellt. Im zweiten Schritt werden Änderungsvorschläge präsentiert. Darunter fallen vor allem drei Punkte: zum einen eine bessere strategische Einbindung von Studierenden in Entscheidungsprozesse - beispielsweise durch eine studentische Prodekanin beziehungsweise einen studentischen Prodekan oder eine studentische Vizepräsidentin beziehungsweise einen studentischen Vizepräsidenten. Zum zweiten ist die Lehre zu nennen, dem Hauptberührungspunkt zwischen der Hochschule und den Studierenden. Um 
hier die Qualität zu verbessern, ist es wichtig, Anreize für gute Lehre zu setzen. Eine Möglichkeit wäre dabei beispielsweise in Berufungsverfahren gute Lehre stärker zu honorieren. Der dritte Vorschlag fällt auch in den Bereich der Lehre. Traditionell ist die Hochschule der Ort der Wissenschaft. Momentan können Studierende allerdings einen Hochschulabschluss erlangen, ohne eine Vorstellung über die Mechanismen des wissenschaftlichen Betriebs zu haben. Eine Lösung dieses Problems wäre die Stärkung von studentischen Forschungsprojekten. Denn nur wer selbst forscht, lernt die Unwägbarkeiten und Herausforderung wissenschaftlicher Arbeit kennen. Abschließend werden die Perspektiven gebündelt.

\section{Das Universitätssystem am Beispiel des Landes- hochschulgesetzes Baden-Württemberg}

Als Studentin beziehungsweise Student besitzt man eine sehr eingeschränkte Sicht auf den Hochschulbetrieb. Oft ist einem nicht bewusst, wie viele Interessenslagen hinter einzelnen Entscheidungen stehen und wie diese Entscheidungen getroffen werden. Deswegen ist es an dieser Stelle notwendig, die verschiedenen Stakeholder und Entscheidungsgremien an der Hochschule darstellen. Hierbei bildet das Landeshochschulgesetz Baden-Württemberg (LHG) die Argumentationsgrundlage.

An den beschlussfassenden Gremien der Hochschule können nach §10 (1) folgende Gruppen teilnehmen: die Hochschullehrerinnen und -lehrer; die akademischen Mitarbeitenden, die Doktoranden, die Studierenden und die sonstigen Mitarbeitenden. Diese bilden die zentralen Stakeholder innerhalb der Hochschule. Die zentralen Organe der Hochschule sind nach \$15 (1) das Rektorat (Präsidium), der Senat und der Hochschulrat. Das Rektorat leitet die Hochschule. Dem Rektorat gehören Rektorin beziehungsweise Rektor, Kanzlerin beziehungsweise Kanzler und weitere Rektoratsmitglieder an, soweit das die Grundordnung vorsieht (\$16 (1)). Das Rektorat ist unter anderem insbesondere für die Struktur- und Entwicklungsplanung einschließlich der Personalentwicklung, die Aufstellung des Entwurfs des Haushaltsvoranschlags und den Vollzug des Haushaltsplans zuständig (§16 (3)). Des Weiteren bereitet das Rektorat nach $§ 16$ (5) die Sitzungen des Senats und seiner Ausschüsse vor und vollzieht deren Beschlüsse sowie die Beschlüsse des Hochschulrats.

Der (akademische) Senat besteht aus dem Rektorat, den Dekaninnen und Dekanen, der Gleichstellungsbeauftragten und höchstens 20 stimmberechtigten 
Mitgliedern. Der Senat entscheidet unter anderem in Angelegenheiten von Forschung, Lehre und Studium. Der Senat ist insbesondere zuständig für die Wahl der haupt- und nebenamtlichen Rektoratsmitglieder, die Zustimmung zum Struktur- und Entwicklungsplan (SEP) und die Stellungnahme zu Entwürfen des Haushaltsvoranschlags oder zum Wirtschaftsplan.

Der Hochschulrat - das dritte Organ der Hochschule - nimmt die strategische Verantwortung der Hochschule wahr. Er kann Beschlussfassung über die Struktur- und Entwicklungspläne und den Haushaltsplan treffen. Er setzt sich aus mindestens sechs und höchstens zwölf Mitgliedern zusammen, die von der Wissenschaftsministerin beziehungsweise dem Wissenschaftsminister bestellt werden. Der Hochschulrat entscheidet unter anderem über die Benennung der Rektorin beziehungsweise des Rektors (vgl. \$16 (6)).

Fakultäten sind die kleinere Organisationseinheit der Hochschule. Mitglieder einer Fakultät sind die Mitglieder des wissenschaftlichen Personals, die Studierenden, die in einem Studiengang eingeschrieben sind, dessen Durchführung der Fakultät obliegt, die immatrikulierten Doktoranden und die sonstigen Mitarbeitenden, die in der Fakultät arbeiten. Die Fakultäten gliedern sich in das Dekanat und den Fakultätsrat. Das Dekanat leitet die Fakultät und ihm gehören der Dekan und die Prodekane an.

Bei den Entscheidungsgremien der Hochschule sind besonders folgende Punkte wichtig: Der Hochschulrat ist das wichtigste Hochschulgremium, dieser setzt die Rektorin beziehungsweise den Rektor ein. Das Rektorat ist für die Erstellung des Struktur- und Entwicklungsplans und des Haushaltes zuständig. Der Senat besitzt die Kompetenz, über die übrigen Rektoratsmitglieder abzustimmen und dem Struktur- und Entwicklungsplan zuzustimmen.

\section{Verbesserungsvorschläge aus studentischer Perspektive}

\section{Strategische Einbindung von Studierenden verbessern}

Bei der Betrachtung des baden-württembergischen Landeshochschulgesetzes hat sich gezeigt, dass sowohl das Rektorat als auch der Hochschulrat zentrale Entscheidungskompetenzen besitzen. So obliegt dem Rektorat das Aufstellen der Haushaltspläne und der Struktur- und Entwicklungspläne. Diese werden zwar dem Senat und dem Hochschulrat vorgelegt, allerdings kann nur der Hochschulrat über Haushaltspläne und den Struktur- und Entwicklungsplan 
Beschlussfassung treffen. Dem Senat bleibt die Möglichkeit, den Struktur- und Entwicklungsplan zuzustimmen und zu den Haushaltsplänen Stellung zu beziehen. Da die Struktur- und Entwicklungspläne langfristig die Agenda der Hochschule bestimmen, ist es wichtig schon früh Studierende in ihren Entstehungsprozess einzubeziehen. Dies ist besonders wichtig, da die Studierenden die Haupttragenden der meisten strategischen Entscheidungen sind.

Gerade hinsichtlich der Digitalisierung sollten Studierende als Hauptnutzendengruppe als integraler Bestandteil zu Wort kommen. Bei dieser Form der Beteiligung sind vor allem zwei Dinge wichtig: Zum einen braucht es engagierte Studierende, die in den Gremien aktiv an der Entwicklung des Struktur- und Entwicklungsplans mithelfen. Hier könnte beispielsweise eine stärkere Einbindung des Senats in die Entwicklung des Struktur- und Entwicklungsplans helfen, da im Senat zwingend Studierende sitzen und Perspektiven der unterschiedlichen Fakultäten eingebracht werden können. Diese Studierenden bringen allerdings konkrete Vorstellungen vom Studium und der Hochschule mit. Diese unterscheiden sich manchmal von den Ansichten Studierender, die nicht in den Gremien sitzen. Um diese ebenfalls in Entscheidungsprozesse einzubinden, bieten sich Evaluationen an. Diese werden an den meisten Hochschulen bezogen auf die Qualität von Lehrveranstaltungen allerdings immer erst zum Semesterende durchgeführt. Solche Befragungen beziehungsweise Bestandsaufnahmen könnten auch von der Hochschulleitung für andere Themenfelder realisiert werden. Hier könnte man mithilfe digitaler Umfragen den bürokratischen Aufwand minimieren. Um für die Studierenden einen Anreiz zu setzen, die Umfrage tatsächlich zu beantworten, könnte diese auf der Frontpage des Learning-Management-Systems erscheinen. Eine Möglichkeit wäre, diese Befragungen ähnlich wie die Rückmeldungen verpflichtend zu machen.

Zum anderen sollten neben der Einbindung in die gesamtstrategische Ausrichtung der Hochschule, Studierende auch auf Fakultätsebene eingebunden sein, was derzeit schon gegeben ist. Analog zu der Gesamtstruktur der Hochschule besitzt das Dekanat sehr viele Kompetenzen, da das Dekanat unter anderem für die Aufstellung des Struktur- und Entwicklungsplans und des Haushaltsplans für die Fakultät zuständig ist. Der Fakultätsrat kann zum Beispiel aus Dekanin beziehungsweise Dekan, weiteren Mitgliedern des Dekanats, bis zu fünf Leiterinnen und Leitern wissenschaftlicher Einrichtungen und gewählten stimmberechtigten Mitgliedern, unter denen sich mindestens drei Studierende befinden müssen, bestehen. Der Fakultätsrat steht dem Dekanat bei der Erstellung des Struktur- und Entwicklungsplans und des Haushaltsplans beratend zur Seite. Da die Evaluationsangelegenheiten dem Dekanat obliegen, kann es sich schon ein breites Bild von der studentischen Perspektive machen. In der Grundordnung der Hochschule ist festgelegt, wie viele Prodekane diese zulässt. 
Hier könnte man über die Einführung einer studentischen Prodekanin beziehungsweise eines studentischen Prodekans nachdenken. Diese würden als vollwertiges Dekanatsmitglied wöchentlich an den Sitzungen teilnehmen und - wie die übrigen Dekanatsmitglieder - durch den Fakultätsrat gewählt. Die Aufgabe würde in der Repräsentation der Studierenden innerhalb des Dekanats bestehen.

Zuletzt soll noch kurz auf die Rolle des Hochschulrats eingegangen werden. Er ist das höchste Gremium der Hochschule und soll die langfristige strategische Ausrichtung der Hochschule bestimmen und die Geschäftsführung des Rektorats überwachen. Der Hochschulrat setzt sich aus hochschulinternen und hochschulexternen Mitgliedern zusammen. Bislang ist ein studentisches Mitglied im Hochschulrat, der vom Wissenschaftsministerium eingesetzt wird, noch keine Pflicht. Dies könnte geändert werden, sodass auch in dem höchsten Hochschulgremium die studentische Perspektive zwangsläufig Gehör findet.

Wie deutlich wurde, geht es aus studentischer Sicht um drei strategische Maßnahmen: Zum einen sollten Studierende stärker in die Produktion der Struktur- und Entwicklungspläne und Haushaltspläne auf Hochschulebene eingebunden werden. Insbesondere sollte regelmäßig ein breites Studierendenfeedback durch digitale Umfragen eingeholt und in die Strategieplanung eingebunden werden. Zum anderen muss über die aktive Einbindung einzelner Studierender, die über eine profunde Kenntnis des Hochschulsystems verfügen, in den Strategieprozess nachgedacht werden. Die strategische Einbindung der Studierenden sollte auch auf Leitungsebene erfolgen. Dabei könnte beispielsweise die Einführung einer studentischen Prodekanin beziehungsweise eines studentischen Prodekans von Nutzen sein. Außerdem sollten auch im Hochschulrat Studierende vertreten sein. Schließlich ist dieser das höchste Gremium der Hochschule.

\section{Der Lehrbetrieb als Kern der Hochschule}

Für die meisten Studierenden besteht der Studienalltag primär aus den Lehrveranstaltungen, die sie besuchen. Selten kommen sie in diesem Zusammenhang mit den oben genannten Entscheidungsorganen oder auch dem Forschungsbetrieb in Kontakt. Umso wichtiger ist es demnach, den Studierenden ein möglichst gutes Lehrangebot $\mathrm{zu}$ unterbreiten. Die Verbesserung der Lehre könnte unter anderem durch drei Maßnahmen umgesetzt werden.

Erstens könnten Studierende aktiver in die Lehre und die Produktion von Lehrmaterialien eingebunden werden. Diese Einbeziehung kann in vielen Bereichen vonstattengehen. Dies könnte bei der Produktion von digitalen Lehrange- 
boten - wie MOOCs - erfolgen. Hier könnten Studierende neben der Produktion beispielsweise auch in der Onlinelehre mit Feedback in Online-Foren und in Blended-Learning-Szenarien einbezogen werden. Dadurch können Studierende viel besser einschätzen, welche Bedürfnisse ihre Kommilitonen haben. Die Studienkommissionen der einzelnen Fächer könnten sich dafür einsetzen, dass es zu einer stärkeren Einbindung der Studierenden in die Produktion der Lehrinhalte und Online-Lehre kommt. Die Umsetzung dessen läge dann allerdings beim Medienzentrum oder einer entsprechenden Einrichtung. Um an diesen Stellen eine bessere Zusammenarbeit zu garantieren, sollte ein regelmäßiger Austausch zwischen Medienzentrum und Studienkommission etabliert werden.

Zweitens kommen aufgrund des deutschen Hochschulsystems Lehrende in ihrer wissenschaftlichen Karriere oft erst spät dazu, lehrend tätig zu werden. Dies macht sich leider auch manchmal in der Lehrqualität bemerkbar. Sinnvoll wäre es, Wissenschaftlerinnen und Wissenschaftler schon früher an den Lehrbetrieb heranzuführen. Des Weiteren sollte die Mühe um gute Lehre honoriert werden. Im baden-württembergischen Landeshochschulgesetz ist in $\S 47$ festgehalten, dass Anwärterinnen beziehungsweise Anwärter auf eine Professur über

pädagogische Eignung, die in der Regel durch Erfahrung in der Lehre oder Ausbildung oder durch Teilnahme an Fort- und Weiterbildungen in Hochschuldidaktik nachzuweisen ist (§47 LHG),

verfügen müssen. Dieser Faktor sollte bei einer Berufung besonders ernst genommen werden. Über Lehrpreise oder die Sichtbarmachung von Bemühungen um gute digitale Lehre könnte ein Anfang gemacht werden. Ein Beispiel hierfür ist die Plattform HFDcert, ein Projekt des Hochschulforums Digitalisierung. Dieses bildet die Bemühung der Lehrenden um digitale Elemente der Lehre durch ePoints ab.

Drittens sollten Studierende selbst über einen Topf verfügen können, mit dem sie Lehrangebote gestalten können. Hiermit können sie Schwerpunkte in der Lehre setzen und technische Geräte für ihr Institut anschaffen. Dadurch wird das Lehrangebot an der Hochschule noch einmal aktiv um die Studierendenperspektive erweitert.

\section{Verzahnung von Forschung und Lehre}

Ein Hochschulstudium sollte Studierende zum wissenschaftlichen Arbeiten befähigen. Dies passiert am ehesten durch eigene Forschungspraxis. Ihr Studium sollte Studierende dazu befähigen, kollaborativ Probleme lösen zu können und 
die Forschungspraxis ihres Faches kennenzulernen. Das könnte man durch zwei Lösungsansätze bewerkstelligen.

Zum einen sollte im Rahmen eines Hochschulstudiums die Abschlussarbeit nicht die erste ernsthafte wissenschaftliche Arbeit sein, die Studierende $\mathrm{zu}$ absolvieren haben. Viel eher könnte beispielsweise das letzte Studienjahr dazu genutzt werden, in Kleingruppen sich einem Thema intensiv zu widmen. Das Ziel einer solchen Arbeit sollte darin bestehen, am Ende des Jahres ein Paper zu veröffentlichen und somit im geschützten Rahmen die Forschungspraxis kennenzulernen. Ein Beispiel für ein solches Lehrforschungsprojekt bietet die Politikwissenschaft der Universität Tübingen.

Zum anderen sollte im Studium allen Studierenden ein Grundverständnis der eigenen Forschungspraxis nähergebracht werden. Daneben sollte es allerdings einzelnen Studierenden, die sich besonders für die Forschung interessieren, möglich gemacht werden, ihr Interesse intensiver zu verfolgen. Hier könnten Studierende beispielsweise selbst Forschungsanträge stellen können.

\section{Fazit}

In diesem Beitrag sollte die studentische Perspektive auf Hochschulentwicklung skizziert werden. Wichtige Forderungen sind vor allem die Einführung einer studentischen Prodekanin beziehungsweise eines studentischen Prodekans, die die studentischen Belange direkt ins Dekanat weitergeben können und eigene studentische Finanzmittel, die Studierende selbst für Neuerungen und Lehrveranstaltungen einsetzen können. 
